\title{
Creating Generalized and Hybrid Set and Library with Neutrosophy and Quad-stage Method
}

\author{
Fu Yuhua
}

\begin{abstract}
Generalized and hybrid set can be created with neutrosophy and quad-stage method. Firstly the generalized and hybrid neutrosophic set is discussed. Based on this, the concepts of "problem set", "solution set", "principle set", "law set", "theory set", "formula set", and the like are presented; Secondly the combination or synthetical body of generalized and hybrid sets is named as "library" (various generalized and hybrid sets can be put into the related "library"); such as "mathematics library", "physics library", "natural science library", "social science library", and the like. As for the constitution of "library", following quad-stage and Chinese ancient "Complete Library of Four Branches of Books", the concept and methodology of a special "Four-library" (including four sub-libraries: information library, question library, correlation library, and achievement library) are proposed. Neutrosophy and quad-stage method can also be used to solve many actual problems within the framework of "set" and "library"; for example, based on the analyses of one "Four-library", jointly solving problem of advance of planet's perihelion with partial results of law of gravity and general relativity (these two theories belong to "gravitational theory set"); and jointly expanding "uncertainty principle" to "certainty-uncertainty principle set" (including three principles in different conditions: "certainty principle", "uncertainty principle", and neutral (fuzzy) "indeterminacy principle") with Heisenberg inequality and Ozawa inequality. Finally, with the help of the concepts of "generalized and hybrid set" and "library", we introduce the concepts of "variational principle of set" and "variational principle of library", and establish a kind of "partial and temporary unified theory of mathematics so far".
\end{abstract}

Key words: Neutrosophy, quad-stage, generalized and hybrid set, neutrosophic set, problem set, solution set, principle set, law set, theory set, formula set, library, Four-library, uncertainty principle, certainty-uncertainty principle set, gravitational theory set, variational principle of set, variational principle of library, partial and temporary unified theory of mathematics so far

Introduction

At present, the concept of "set" has failed to meet the need to solve many practical problems, therefore it should be expanded into generalized and hybrid set. There are many ways to create generalized and hybrid set. This paper discusses the application of neutrosophy and quad-stage method to create generalized and hybrid set, and focuses on generalized and hybrid neutrosophic set. On this basis, some new concepts and methods related to generalized and hybrid set are presented.

\section{Basic Contents of Neutrosophy}

Neutrosophy is proposed by Prof. Florentin Smarandache in 1995.

Neutrosophy is a new branch of philosophy that studies the origin, nature, and scope of neutralities, as well as their interactions with different ideational spectra.

This theory considers every notion or idea $<A>$ together with its opposite or negation $<$ Anti-A $>$ and the spectrum of "neutralities" $<$ Neut-A $>$ (i.e. notions or ideas located between 
the two extremes, supporting neither $<$ A $>$ nor $<$ Anti-A $>$ ). The $<$ Neut-A $>$ and $<$ Anti-A $>$ ideas together are referred to as $<$ Non-A $>$.

Neutrosophy is the base of neutrosophic logic, neutrosophic set, neutrosophic probability and statistics used in engineering applications (especially for software and information fusion), medicine, military, cybernetics, and physics.

Neutrosophic Logic is a general framework for unification of many existing logics, such as fuzzy logic (especially intuitionistic fuzzy logic), paraconsistent logic, intuitionistic logic, etc. The main idea of NL is to characterize each logical statement in a 3D Neutrosophic Space, where each dimension of the space represents respectively the truth $(T)$, the falsehood $(F)$, and the indeterminacy (I) of the statement under consideration, where $T, I$, $F$ are standard or non-standard real subsets of ]-0, 1+[ without necessarily connection between them.

More information about Neutrosophy can be found in references [1, 2].

\section{Basic Contents of Quad-stage}

Quad-stage (Four stages) is presented in reference [3], it is the expansion of Hegel's triad-stage (triad thesis, antithesis, synthesis of development). The four stages are "general theses", "general antitheses", "the most important and the most complicated universal relations", and "general syntheses". They can be stated as follows.

The first stage, for the beginning of development (thesis), the thesis should be widely, deeply, carefully and repeatedly contacted, explored, analyzed, perfected and so on; this is the stage of general theses. It should be noted that, here the thesis will be evolved into two or three, even more theses step by step. In addition, if in other stage we find that the first stage's work is not yet completed, then we may come back to do some additional work for the first stage.

The second stage, for the appearance of opposite (antithesis), the antithesis should be also widely, deeply, carefully and repeatedly contacted, explored, analyzed, perfected and so on; this is the stage of general antitheses. It should be also noted that, here the antithesis will be evolved into two or three, even more antitheses step by step.

The third stage is the one that the most important and the most complicated universal relations, namely the seedtime inherited from the past and carried on for the future. Its purpose is to establish the universal relations in the widest scope. This widest scope contains all the regions related and non-related to the "general theses", "general antitheses", and the like. This stage's foundational works are to contact, grasp, discover, dig, and even create the opportunities, pieces of information, and so on as many as possible. The degree of the universal relations may be different, theoretically its upper limit is to connect all the existences, pieces of information and so on related to matters, spirits and so on in the universe; for the cases such as to create science fiction, even may connect all the existences, pieces of information and so on in the virtual world. Obviously, this stage provides all possibilities to fully use the complete achievements of nature and society, as well as all the humanity's wisdoms in the past, present and future. Therefore this stage is shortened as "universal relations" (for other stages, the universal relations are also existed, but their importance and complexity cannot be compared with the ones in this stage).

The fourth stage, to carry on the unification and synthesis regarding various opposites and the suitable pieces of information, factors, and so on; and reach one or more results which are the best or agreed with some conditions; this is the stage of "general syntheses". The results of this stage are called "synthesized second generation theses", all or partial of them may become the beginning of the next quad-stage. 
In quad-stage method, "general theses" may be considered as the notion or idea $<A>$; "general antitheses" may be considered as the notion or idea <Anti-A>; "the most important and the most complicated universal relations" may be considered as the notion or idea $<$ Neut-A>; and "general syntheses" are the final results.

The different kinds of results in the above mentioned four stages can also be classified and induced with the viewpoints of neutrosophy. Thus, the theory and achievement of neutrosophy can be applied as many as possible, and the method of quad-stage will be more effective.

\section{Creating Generalized and Hybrid Neutrosophic Set with Neutrosophy and Quad-stage Method}

Firstly we consider the general meaning's generalized and hybrid neutrosophic set (it may have nothing to do with quad-stage).

The ordinary neutrosophic set generally includes three elements: element $\langle A\rangle$, its opposite element $<$ Anti-A $>$, and element $<$ Neut-A $>$ (i.e. element located between the two extremes, supporting neither $<A>$ nor $<A n t i-A>$ ); While for generalized and hybrid neutrosophic set, besides $\langle A\rangle,\langle$ Anti-A $>$, and $\langle$ Neut-A $>$, considering to combine other element, such as element $\langle B\rangle,\langle\mathrm{C}\rangle,\langle\mathrm{D}\rangle$ and the like, (they may belong to Fuzzy Sets, Rough sets, Soft Sets, Genuine sets, and the like). For example, for a problem of colors, supposing that an ordinary neutrosophic set contains three colors: black, white, and grey; then the corresponding generalized and hybrid neutrosophic set may also contain the colors of red, yellow, green, and the like, even other elements such as picture, sound, and the like that are related or non-related to colors.

In quad-stage, some types of generalized and hybrid neutrosophic sets can be defined (created) as follows.

If all elements $(<A>,<$ Anti-A $>,<$ Neut-A $>$, as well as $\langle B>$ and the like) are located at the same stage in the method of quad-stage, then they can be defined as the first type of generalized and hybrid neutrosophic sets. For more detail, if all elements are located at the first stage, then they can be defined as the first type of generalized and hybrid neutrosophic sets in first stage; similarly, the first type of generalized and hybrid neutrosophic sets in second stage, in third stage, and in fourth stage can be defined respectively.

If all elements $(<A>,<$ Anti-A $>,<$ Neut-A $>$, as well as $<B>$ and the like) are located at the different stages in the method of quad-stage, then they can be defined as the second type of generalized and hybrid neutrosophic sets. For more detail, if all elements are located at the first stage and second stage, then they can be defined as the second type of generalized and hybrid neutrosophic sets in first stage and second stage; similarly, the second type of generalized and hybrid neutrosophic sets in other stages can be defined.

In addition, besides the concept of element, considering that in some cases several principles have to be combined to solve a problem, or meet other requirements (such as collecting the results or achievements), then the concept of "principle set" can be proposed (it belongs to generalized and hybrid neutrosophic sets). Similarly, other concepts of generalized and hybrid neutrosophic sets can also be defined, for example, "problem set", "solution set", "law set", "formula set", and their combination such as "principle, law and formula set", and the like.

For quad-stage, for the reason that there are all kinds of problems in four stages from beginning to end, so the "problem set" in quad-stage can be established. According to the viewpoint of neutrosophy, the "problem set" can include: problems appeared in the past, problems appeared at present, and problems appeared in the future; it can also include: resolved problems, unresolved problems, and partially resolved problems. 
In four stages, many problems can be solved continually, therefore the "solution set" in quad-stage can be established. According to the viewpoint of neutrosophy, the "solution set" can include: solutions appeared in the past, solutions appeared at present, and solutions appeared in the future; it can also include: published solutions, non-published solutions, and partially published solutions.

Now we give several examples of "solution set". For "Pythagorean theorem", there have been about 500 solutions; for "gravitational problem", there have been many solutions to solve it, such as the solution given by law of gravity and the solution given by general relativity; in the medical field, there have been Western medicine, Chinese medicine, combination of Chinese medicine and Western medicine, and the like.

An example of "theory set" is "gravitational theory set" (including law of gravity, general relativity, and the like)

\section{Concept of "Library" and application of "Four-library"}

For the reason that the limitation of the concept of "set", also in order to better play the role of "set" (including generalized and hybrid set), we introduce the concept of "library".

The combination or synthetical body of generalized and hybrid sets is named as "library", it can contain various "elements", "sets", "informations", "processes", "correlations", "problems", "achievements", and the like.

There are many ways to run the classification of "library". According to the scale, all of the libraries can be divided into "small library", "medium library", and "big library". For example, "Euclidean geometry library" and "Newtonian mechanics library" belong to "small library", "Mathematics library" and "Physics library" belong to "medium library", and "Natural science library" and "Social science library" belong to "big library". In addition, according to the number of "sub-library" (sometimes "sub-library" is also shortened as "library"), it can be named as "Single-library" ("One-library", namely "library of one sublibrary"), "Two-library" ("Double-library", namely "library of two sub-libraries"), "Threelibrary", "Four-library", and the like. While in this paper we will focus on "Four-library".

Following quad-stage and Chinese ancient "Complete Library of Four Branches of Books", for some field, event, dynasty, period, discipline, theory, and their combination (such as one dynasty in a special period), the concept and methodology of a special "Fourlibrary" (including four sub-libraries: information library, question library, correlation library, and achievement library) are proposed. In which, information library mainly corresponds to the contents of the first stage of quad-stage; question library mainly corresponds to the contents of the second stage of quad-stage; correlation library mainly corresponds to the third stage of quad-stage; and achievement library mainly corresponds to the content of the fourth stage of quad-stage. However, it is different from the quad-stage, information library can be considered as a "total-library", and question library, correlation library, and achievement library can be considered as "sub-library". The "total-library" may include all or part of the contents (such as directory, abstracts, etc) of sub-libraries. Of course, generally the "total-library" includes part of the contents of sub-libraries only.

Due to the existence of "total-library" and "sub-library", besides "Four-library", we can also present the concepts of "One-library", "Two-library", "Three-library", "Five-library", "Six-library", and the like. However, for obvious reason, many people are not interested in "Thirteen-library".

As the applications of "Four-library", we will discuss jointly solving problem of advance of planet's perihelion with partial results of law of gravity and partial results of general relativity (these two theories belong to "gravitational theory set"; their total contents can be put into achievement library, and partial contents can be put into information library); and jointly expanding "uncertainty principle" to "certainty-uncertainty principle set" (including 
three principles in different conditions: "certainty principle", "uncertainty principle", and neutral (fuzzy) "indeterminacy principle") with Heisenberg inequality and Ozawa inequality.

For the "event" of "advance of planet's perihelion", or "library of advance of planet's perihelion", its corresponding "Four-library" (including information library, question library, correlation library, and achievement library) can be established. According to the results of information library and achievement library we can see that: in order to describe the motion of the planet, the main bases are law of gravity and general relativity. According to the question library we can see that: the elliptical orbit given by law of gravity cannot provide the value of advance of planet's perihelion, while the value of advance given by general relativity is small different from the accurate astronomical observation. According to the correlation library we can see that: some scholars believe that the vortex motion is existed in solar system. On these bases, a new explanation for advance of planet's perihelion can be given, and the result can be put into the achievement library.

In the past, only few people consider that solving the same problem with Newton's law of gravity and Einstein's general relativity simultaneously. However, the concept of "Fourlibrary" presents such idea and method: Newton's law of gravity and Einstein's general relativity can be used to solve the same problem simultaneously. In this regard, many specific ideas can be given. The simplest way is: to combine partial result of Newton's law of gravity and partial result of Einstein's general relativity, and form a new result. In reference [4], applying this way that a new explanation is presented: the advance of planetary perihelion is the combined result of two motions. The first elliptical motion creates the perihelion, and the second vortex motion creates the advance of perihelion. In the motion of planet-sun system, under the action of gravity, the planetary orbit is a closed ellipse, and consistent with the law of conservation of energy. Meanwhile, the planet also participates in the vortex motion of solar system taking the sun as center; the long-term trend of the vortex is the further topic to be solved, but in the short-term may be considered that due to the inertia the planetary perihelion will run circular motion in vortex and lead to the advance of perihelion, thus also without acting against the law of conservation of energy. Based on the result of general relativity, the approximate angular velocity of advance of perihelion is given; based on accurate astronomical observation, the accurate angular velocity is given. Finally the approximate expression for circular velocity of solar system's vortex motion is presented. For ordinary vortex motion the circular velocity is inversely proportional to the radius r, but for solar system's vortex motion, it is inversely proportional to $r^{3 / 2}$.

Now we give the solving process.

In the planet-sun system, taking the solar center as the origin of coordinate, according to Newton's law of gravity, the planetary orbit equation reads

$$
\frac{(x-k)^{2}}{a^{2}}+\frac{y^{2}}{b^{2}}=1
$$

where: $k$ is the semi-focal length of ellipse.

According to the rotate transformation in Cartesian coordinate system, it gives

$$
\begin{aligned}
& x=x^{\prime} \cos \theta-y^{\prime} \sin \theta \\
& y=x^{\prime} \sin \theta+y^{\prime} \cos \theta
\end{aligned}
$$

where: $\theta$ is the angle of rotation (namely the angle of advance), according to calculated value or accurate astronomical observation, $\theta=\omega t$ or $\theta=\omega^{\prime} t$. follows

Thus, after considering the vortex motion, the planetary rotation orbit equation is as 


$$
\frac{\left(x^{\prime} \cos \theta-y^{\prime} \sin \theta-k\right)^{2}}{a^{2}}+\frac{\left(x^{\prime} \sin \theta+y^{\prime} \cos \theta\right)^{2}}{b^{2}}=1
$$

The value of $\theta$ can be given as follows.

According to general relativity, the value of advance of planetary perihelion reads

$$
\varepsilon=\frac{24 \pi^{3} a^{2}}{T^{2} c^{2}\left(1-e^{2}\right)}
$$

where: $\mathrm{c}$ is the speed of light; $\mathrm{T}, \mathrm{a}$, and e are orbital period, semi-major axis and eccentricity respectively. follows

Taking the sun as center, the angular velocity of advance of planetary perihelion is as

$$
\omega=\frac{\varepsilon}{T}=\frac{24 \pi^{3} a^{2}}{T^{3} c^{2}\left(1-e^{2}\right)}
$$

According to Kepler's third law, it gives

$$
\frac{T^{2}}{a^{3}}=\frac{4 \pi^{2}}{G M}
$$

where: $\mathrm{G}$ is the gravitational constant, and $\mathrm{M}$ is the solar mass.

Then it gives

$$
\omega=\frac{3 G^{3 / 2} M^{3 / 2}}{a^{5 / 2} c^{2}\left(1-e^{2}\right)}
$$

According to this expression we can see that, the angular velocity of advance of planetary perihelion is inversely proportional to $a^{5 / 2}$.

For the value of general relativity, there is small difference compared with accurate astronomical observation, therefore the accurate angular velocity of advance of perihelion is as follows.

$$
\omega^{\prime}=\frac{\varepsilon^{\prime}}{T}
$$

where: $\varepsilon^{\prime}$ is the accurate astronomical observation of advance of perihelion.

Now we discuss the circular velocity of this vortex motion at the position of radius $r$.

Assuming that the angular velocity of solar system's vortex motion is approximately equal to the angular velocity of advance of planetary perihelion, and in Eq.(3) the value of $a$ is replaced by the radius $r$, moreover the eccentricity $e$ is omitted, then apply the formula $v=r \omega$, it gives the circular velocity of this vortex motion at the position of radius $r$ as follows

$$
v \approx \frac{3 G^{3 / 2} M^{3 / 2}}{r^{3 / 2} c^{2}}
$$


From this expression we can see that, unlike the ordinary vortex motion (its circular velocity is inversely proportional to the radius $r$ ), for solar system's vortex motion, the circular velocity is inversely proportional to $r^{3 / 2}$.

Now we discuss the problem of uncertainty principle.

For this "theory" of "uncertainty principle", its "Four-library" (including information library, question library, correlation library, and achievement library) can be established. According to the information library and achievement library we can see that: "uncertainty principle" has been widely used in physics. According to the question library we can see that: some scholars believe that "uncertainty principle" is incorrect. According to the correlation library we can see that: there is contradiction between Heisenberg inequality and Ozawa inequality. On these bases, Heisenberg inequality and Ozawa inequality can be used to jointly expand "uncertainty principle" to "certainty-uncertainty principle set" (according to classification of neutrosophy, this set includs three principles in different conditions: "certainty principle", namely a particle's position and momentum can be known simultaneously; "uncertainty principle", namely a particle's position and momentum cannot be known simultaneously; and neutral (fuzzy) "indeterminacy principle", namely whether or not a particle's position and momentum can be known simultaneously is undetermined. This result can be put into the achievement library.

Firstly we discuss the two methods to expand "uncertainty principle" to "certaintyuncertainty principle set" with Heisenberg inequality and Ozawa inequality respectively.

In quantum mechanics, the uncertainty principle refers to the position and momentum of a particle cannot be determined simultaneously, the uncertainty of position $(\Delta x)$ and uncertainty of momentum ( $\Delta p$ ) obey the following Heisenberg inequality

$$
\Delta x \Delta p \geq h / 4 \pi
$$

where, $h$ is the Planck constant.

Heisenberg inequality (Eq.3) can be changed into the following form of equality

$$
\Delta x \Delta p=k h / 4 \pi
$$

where, $k$ is a real number and $k \geq 1$.

Ozawa inequality ${ }^{[5]}$ can be written as follows

$$
\Delta Q \Delta P+\Delta Q \sigma(P)+\sigma(Q) \Delta P \geq h / 4 \pi
$$

It can be changed into the following form of equality

$$
\Delta Q \Delta P+\Delta Q \sigma(P)+\sigma(Q) \Delta P=k h / 4 \pi
$$

where, $k$ is a real number and $k \geq 1$.

According to Neutrosophy, any proposition has three situations of truth, falsehood and indeterminacy respectively. With the help of Heisenberg inequality, the original uncertainty principle can be extended into the following Heisenberg type's "certainty-uncertainty principle set"

$$
\Delta x \Delta p=K h
$$

where, $K$ is a real number and $K>0$. 


\section{Eq.(7) can be divided into three principles:}

The first one is the "uncertainty principle" $\left(K \geq K_{1}\right)$ : a particle's position and momentum cannot be known simultaneously.

Obviously, if $K_{1}=1 / 4 \pi$, then it is the original uncertainty principle.

The second one is the "certainty principle" $\left(K \leq K_{2}\right)$ : a particle's position and momentum can be known simultaneously.

Referring to the experiments for establishing Ozawa inequality, the value of $K_{2}$ can be decided by related experiments.

The third one is the neutral (fuzzy) "indeterminacy principle" ( $K_{2}<K<K_{1}$ ): whether or not a particle's position and momentum can be known simultaneously is undetermined.

Similarly, the original Ozawa inequality can be extended into the following Ozawa type's "certainty-uncertainty principle set"

$$
\Delta Q \Delta P+\Delta Q \sigma(P)+\sigma(Q) \Delta P=K h
$$

where, $K$ is a real number and $K>0$.

Eq.(8) can be divided into three principles:

The first one is the "certainty principle" ( $K \geq K_{1}$ ): a particle's position and momentum can be known (namely can be measured with zero-error) simultaneously (here $\sigma(P)$ or $\sigma(Q)$ is equal to infinity).

Obviously, if $K_{1}=1 / 4 \pi$, then it is the original Ozawa inequality (with equality form).

It should be noted that here the first one is not the uncertainty principle, but certainty principle.

The second one is the "uncertainty principle" $\left(K \leq K_{2}\right)$ : a particle's position and momentum cannot be known simultaneously.

The third one is the neutral (fuzzy) "indeterminacy principle" ( $K_{2}<K<K_{1}$ ): whether or not a particle's position and momentum can be known simultaneously is undetermined.

On this basis, we can discuss the way to expand "uncertainty principle" to HeisenbergOzawa type's "certainty-uncertainty principle set" with Heisenberg inequality and Ozawa inequality jointly.

Supposing that Heisenberg inequality is suitable in domain $\Omega_{1}$, Ozawa inequality is suitable in domain $\Omega_{2}$, and the remained domain is $\Omega_{3}$. The Heisenberg-Ozawa type's "certainty-uncertainty principle set" can be divided into three principles:

The first one is the "uncertainty principle" (it is suitable in domain $\Omega_{1}$ ): a particle's position and momentum cannot be known simultaneously.

The second one is the "certainty principle" (it is suitable in domain $\Omega_{2}$ ): a particle's position and momentum can be known simultaneously. 
The third one is the neutral (fuzzy) "indeterminacy principle" (it is suitable in domain $\Omega_{3}$ ): whether or not a particle's position and momentum can be known simultaneously is undetermined.

We already discuss the concept of "library" and the application of "Four-library". It should be noted that, in some cases, the term of "library" can be replaced by other terms, such as "department", "world", "universe", and the like.

5 A kind of "partial and temporary unified theory of mathematics so far"

Now, with the help of the concepts of "generalized and hybrid set" and "library", we introduce the concepts of "variational principle of set" and "variational principle of library", and establish a kind of "partial and temporary unified theory of mathematics so far".

In reference [2], for unified dealing with the problems of natural science, applying least square method, "partial and temporary unified theory of natural science so far" can be expressed in the following form of "partial and temporary unified variational principle of natural science so far"

$$
\Pi_{\mathrm{NATURE}}=\sum_{1}^{n} W_{i} \int_{\Omega_{i}} F_{i}^{2} d \Omega_{i}+\sum_{1}^{m} W_{j}^{\prime} S_{j}^{2}=\min _{0}
$$

where: $\min _{0}$ was introduced in reference [6], indicating the minimum and its value should be equal to zero, the subscript NATURE denotes that the suitable scope is all of the problems of natural science, all of the equations $F_{i}=0$ denote so far discovered (derived) all of the equations related to natural science (their suitable scopes are $\Omega_{i}$ ), all of the equations $S_{i}=0$ denote so far discovered (derived) all of the solitary equations related to natural science (they are suitable on solitary points or in some special cases), and $W_{i}$ and $W_{j}{ }^{\prime}$ are suitable positive weighted constants.

In this way, the theory of everything to express all of natural laws, described by Hawking that a single equation could be written on a T-shirt, is partially and temporarily realized in the form of "partial and temporary unified variational principle of natural science so far".

However, the disadvantages of such a "partial and temporary unified theory of natural science so far" are also very obvious, namely it is disorganized and lack of layers.

In order to avoid this disadvantage, we introduce some concepts.

The first one is the concept of "variational principle of set".

The meaning of "variational principle of set" is the variational principle formed by using least square method (LSM) to process all equations and equalities included in a set (including generalized and hybrid set).

An example to form "variational principle of set of quadratic equation of one unknown" is given below.

In "set of quadratic equation of one unknown", the main equations and equalities related to quadratic equation of one unknown are as follows.

The Standard form of quadratic equation of one unknown reads

$$
F_{1}=0
$$


where: $F_{1}=a x^{2}+b x+c$

Two root formulas are as follows

$$
F_{2}=0
$$

where: $F_{2}=x_{1}-\frac{-b+\sqrt{b^{2}-4 a c}}{2 a}$

And

$$
F_{3}=0
$$

where: $F_{3}=x_{2}-\frac{-b-\sqrt{b^{2}-4 a c}}{2 a}$

Due to the existence of complex solution, the formulas related to complex should also be considered, among which the most famous one is Euler's formula

$$
F_{4}=0
$$

where: $F_{4}=e^{\pi i}+1$

Two important equalities related to Euler's formula are as follows

$$
F_{5}=0
$$

where: $F_{5}=\pi-3.14159265 .$.

And

$$
F_{6}=0
$$

where: $F_{6}=e-2.718281828$.

And so forth.

By using least square method (LSM) to process the equations and equalities related to "set of quadratic equation of one unknown", we have the following "variational principle of set of quadratic equation of one unknown"

$$
\Pi_{\text {QEOOUset }}=\Pi_{1}+\Pi_{2}+\Pi_{3}+\cdots=\min _{0}
$$

where: the subscript QEOOUset denotes "set of quadratic equation of one unknown", $\Pi_{1}=F_{1}^{2}, \Pi_{2}=F_{2}^{2}, \Pi_{3}=F_{3}^{2}, \ldots$

Similarly, for "set of cubic equation of one unknown", we have "variational principle of set of cubic equation of one unknown" $\left(\Pi_{\text {CEOOUset }}=\min _{0}\right)$; for "set of quartic equation of one unknown", we have "variational principle of set of quartic equation of one unknown" $\left(\Pi_{\mathrm{Q}^{\prime} \text { EOOUset }}=\min _{0}\right)$; and the like.

The second one is the concept of "variational principle of library". 
For the reason that the combination or synthetical body of generalized and hybrid sets is named as "library" (various generalized and hybrid sets can be put into the related "library"), therefore the combination or synthetical body of "variational principle of set" will form "variational principle of library".

For example, if the combination or synthetical body of "set of quadratic equation of one unknown", "set of cubic equation of one unknown", "set of quartic equation of one unknown", and the like is named as "library of equation of degree n with one unknown", then the expression of "variational principle of library of equation of degree $n$ with one unknown" is as follows

$$
\Pi_{\text {EDNOUlibrary }}=\Pi_{\text {QEOOUset }}+\Pi_{\text {CEOOUset }}+\Pi_{\mathrm{Q}^{\prime} \text { EOOUset }}+\cdots=\min _{0}
$$

After defining "variational principle of set" and "variational principle of library", "partial and temporary unified theory of natural science so far" proposed in reference [2], namely Eq.(9), can be re-organized and expressed by the combination or synthetical body of "variational principle of set" and/or "variational principle of library".

"partial and temporary unified theory of natural science so far" expressed by the combination or synthetical body of "variational principle of set" is as follows

$$
\Pi_{\text {NATURElibry }}=\Pi_{\text {MATHset }}+\Pi_{\text {PHYSset }}+\Pi_{\text {CHEMset }}+\cdots=\min _{0}
$$

where: $\Pi_{\text {MATHset }}$ is "variational principle of mathematics set", $\Pi_{\text {PHYSset }}$ is "variational principle of physics set", $\Pi_{\text {CHEMset }}$ is "variational principle of chemistry set".

"partial and temporary unified theory of natural science so far" expressed by the combination or synthetical body of "variational principle of library (sub-library)" is as follows

$$
\Pi_{\text {NATURElibary }}=\Pi_{\text {MATHsublibary }}+\Pi_{\text {PHYSsublibary }}+\Pi_{\text {CHEMsublibrary }}+\cdots=\min _{0}
$$

where , $\Pi_{\text {MATHsublibary }}$ is "variational principle of mathematics library (sub-library)", $\Pi_{\text {PHYSsublitary }}$ is "variational principle of physics library (sub-library)", $\Pi_{\text {CHEMsublitrary }}$ is "variational principle of chemistry library (sub-library)".

From Eq.(10) and Eq.(11) we can see that, there is not strict boundary between "variational principle of set" and "variational principle of library", for the same content, not only it can be named as "variational principle of set", but also can be named as "variational principle of library".

In addition, for the division of layers, all kinds of "sub-set" can be divided into "first order sub-set", "second order sub-set" and the like; all kinds of "sub-library" can be divided into "first order sub-library", "second order sub-library" and the like. Accordingly, all kinds of "variational principle of sub-set" can be divided into "first order variational principle of sub-set", "second order variational principle of sub-set" and the like; all kinds of "variational principle of sub-library" can be divided into "first order variational principle of sub-library", "second order variational principle of sub-library" and the like.

For example, in Eq.(11), if "mathematics library" can be considered as "first order (mathematics) sub-library", then according to neutrosophy it can be divided into three "second order sub-library": "second order elementary mathematics sub-library", "second order secondary mathematics sub-library", and "second order higher mathematics sublibrary"; While, "second order elementary mathematics sub-library" can be divided into: "third order elementary geometry sub-library", "third order elementary algebra sub-library", "third order elementary trigonometry sub-library", and the like. 
It should also be noted that, after re-organized "partial and temporary unified theory of natural science so far" (in which we already apply neutrosophy and quad-stage method), and reach Eq.(10) and Eq. (11), we can also apply neutrosophy and quad-stage method to re-organize Eq.(10) and Eq. (11) again. For example, we can apply the concept and method of "Four-library" to re-organize all of the first order sub-libraries into "First order Four-library", all of the second order sub-libraries into "Second order Four-library", and the like.

Now we discuss the application of "set" and "library", as well as "variational principle of set" and "variational principle of library".

As dealing with a specific problem, we can select some contents included in related "set" and "library", as well as "variational principle of set" and "variational principle of library", and form a simplified variational principle. Based on this simplified variational principle, the accurate solution or approximate solution can be reached.

For example, as solving the problem of advance of planetary perihelion, the elliptical orbit (namely Eq.(1)) can be selected in "library of law of gravity"; the formulas of rotate transformation in Cartesian coordinate system can be selected in "formula set of transformation in Cartesian coordinate system"; and the following variational principle can be formed

$$
\Pi=\Pi_{1}+\Pi_{2}=\min _{0}
$$

where, $\Pi_{1}=\left(\frac{(x-k)^{2}}{a^{2}}+\frac{y^{2}}{b^{2}}-1\right)^{2}$

$$
\Pi_{2}=\left(x-x^{\prime} \cos \theta+y^{\prime} \sin \theta\right)^{2}+\left(y-x^{\prime} \sin \theta-y^{\prime} \cos \theta\right)^{2}
$$

While the accurate solution of Eq.(12) is Eq.(2).

As for the example to find approximate solution with optimization method, see reference [6], in which the best approximate solution was given for problem of advance of Mercury's perihelion

\section{Conclusions}

By using neutrosophy and quad-stage method, generalized and hybrid set can be created, whose example is creating generalized and hybrid neutrosophic set. On this basis, the concepts of "problem set", "solution set", "principle set", "law set", "theory set", "formula set", and the like are presented; and then the concept of "library" is discussed. For the constitution of "library", the standard model is not existed (various generalized and hybrid sets can be put into the related "library"). According to the requirements and the existing conditions can create various sorts of "library". As the first example of "library", following quad-stage and Chinese ancient "Complete Library of Four Branches of Books", the concept and methodology of a special "Four-library" (including four sub-libraries: information library, question library, correlation library, and achievement library) are proposed. Examples in this paper show that the applications of "Four-library" can effectively solve many complex problems, so it has good prospects. Finally, with the help of the concepts of "generalized and hybrid set" and "library", we introduce the concepts of "variational principle of set" and "variational principle of library", and establish a kind of "partial and temporary unified theory of mathematics so far"; in which, it is the first time to put all of mathematics formulas into a multi-layer "variational principle of library". 


\section{References}

1 Florentin Smarandache, A Unifying Field in Logics: Neutrosophic Logic. Neutrosophy, Neutrosophic Set, Neutrosophic Probability and Statistics, third edition, Xiquan, Phoenix, 2003

2 Fu Yuhua, Neutrosophic Examples in Physics, Neutrosophic Sets and Systems, Vol. 1, 2013

3 Fu Yuhua, Quad general theses, general antitheses, universal relations, general syntheses in development -Expansion of Hegelian triad thesis, antithesis, synthesis, Matter Regularity, No.1, 2011, 61-64

4 Fu Yuhua. New Explanation of Advance of Planetary Perihelion and Solar System's Vortex Motion. See: Unsolved Problems in Special and General Relativity. Edited by: Florentin Smarandache, Fu Yuhua and Zhao Fengjuan. Education Publishing, 2013. 249252

5 Masanao Ozawa, Physical content of Heisenberg's uncertainty relation: limitation and reformulation, Physics Letters A 318 (2003), 21-29

$6 \mathrm{Fu}$ Yuhua, New solution for problem of advance of Mercury's perihelion, Acta Astronomica Sinica, No.4, 1989 